\title{
Measuring spatial vibration using continuous laser scanning ${ }^{1}$
}

\author{
Izhak Bucher and Oded Wertheim \\ Faculty of Mechanical Engineering, Technion, Haifa \\ 32000, Israel
}

\section{Received 10 April 2000 \\ Revised 10 April 2000}

This paper presents a method, which allows one to use a single point laser vibrometer as a continuous sensor measuring along a line or a 2D surface. The mathematical background of the curve-fitting procedure and the necessary signal processing allowing one to extract the amplitude of sinusoidal vibration are discussed. In the current work, use has been made with an ordinary laser interferometer equipped with glavanometerbased $\mathrm{x}$, y mirros. This system is not designed for continuous scanning therefore some effort needs to be spent in order to overcome the dynamical characteristics of this system. The potential of such an instrument, as demonstrated in this work, may encourage the development of mechanically better scanning devices.

\section{Introduction}

Laser interferometers are traditionally being used as single point sensors. Since one wishes to obtain the spatial as well as temporal information about a vibrating surface, the information is collected by discretely stepping through a grid of points marked on the structure. In the discrete approach, the measuring beam is being moved every time to one of many pre-selected grid points on the tested structure and for each measured point, the response needs to be acquired and curvefitted, mostly using fft-based methods. This process is time-consuming and the obtained spatial resolution

\footnotetext{
${ }^{1}$ An earlier form of this paper was presented at the 1 st International Conference on Vibration Measurements by Laser Techniques, Ancona, Italy, October 1994. The conference was organized by the Italian Association of Laser Velocimetry. The articles were selected, edited, and reviewed by a committee chaired by: Professor Enrico Primo Tomasini, Department of Mechanics, Faculty of Engineering, University of Ancon, Italy.
}

depends on the density of the grid and on DFT-related problems (i.e. leakage). Continuous scanning on the other hand can be used to theoretically achieve an infinite spatial resolution. In order to achieve a good spatial description of the operating mode shapes, all the participating components of the instantaneous measured velocity signal should be understood and included in a suitable parametric model. In this paper such a parametisation in conjunction with an efficient and accurate curve fitting method is described. The presented method can accommodate generally shaped periodic scanning trajectories and can also accommodate nonsmooth vibrating shapes using an arbitrary selection of basis function to describe the spatial response.

\section{Mathematical background}

Consider a surface vibrating harmonically so that its motion can be mathematically described as:

$$
v(x, y, t)=A(x, y) \sin (\omega t+\phi)
$$

Assuming that the amplitude function $A(x, y)$ can be approximated sufficiently well by a linear combination of some pre-selected basis functions- $f_{k}(x, y)$, one may write:

$$
A(x, y) \approx \sum_{k=1}^{P} a_{k} f_{k}(x, y)
$$

where $a_{k}$ are some coefficients.

Given a continuous scanning trajectory, which describes the position of the measuring laser-beam in the $\mathrm{x}-\mathrm{y}$ plane as a parametric function of time, i.e.

$$
x=\eta(t), \quad y=\mu(t)
$$

One can observe that due to the scanning operation, the observed amplitude function- $A(x, y)$ becomes a function of time (modulated by the motion of the laser-beam):

$$
\begin{aligned}
A(x, y) & =A(\eta(t), \mu(t)) \\
& \approx \sum_{k=1}^{P} a_{k} f_{k}(\eta(t), \mu(t))
\end{aligned}
$$


As will be shown below, continuous scanning allows one to curve-fit the coefficients $\left(a_{k}\right)$ in order to obtain the (sought) two-dimensional amplitude function. This will be illustrated by an example of one- and two-dimensional scanning.

\subsection{One-dimensional scanning}

Assuming that the laser is performing a sinusoidal scanning along the $\mathrm{x}$-axis and that the measuring laserbeam is always perpendicular to the vibrating surface, we are able to write for this case:

$$
\begin{aligned}
& x=\eta(t)=X_{0} \sin \left(\Omega t+\theta_{x}\right) \\
& y=Y_{0}=\text { const. }
\end{aligned}
$$

The measured response (voltage) can be expressed in terms of some basis functions as:

$$
\begin{gathered}
V(t) \approx\left(\sum_{k=1}^{P} a_{k} f_{k}\left(X_{0} \sin \left(\Omega t+\theta_{x}\right)\right)\right) \\
\sin (\omega t+\phi)
\end{gathered}
$$

where we have dropped the dependence upon $y$ in this case.

Choosing, for example, $f_{k}(x)=x^{k-1}$, it can be shown that:

$$
\begin{aligned}
V(t) \approx & \left(a_{1}+a_{2} X_{0} \sin \left(\omega t+\theta_{x}\right)\right. \\
& \left.+a_{3}\left(X_{0} \sin \left(\Omega t+\theta_{x}\right)\right)^{2}+\ldots\right) \\
& \sin (\omega t+\phi)
\end{aligned}
$$

Expanding Eq. (6a) using standard trigonometric identities, we can observe that each coefficient is multiplied by functions containing several harmonics. Expanding Eq. (6a) and using some trigonometric identities we are able to isolate the coefficients of the sought parameters:

$$
\begin{aligned}
a_{1}: & \sin (\omega t+\phi) \\
a_{2}: & \frac{1}{2} X_{0}\left(\cos \left((\Omega-\omega) t+\theta_{x}-\phi\right)\right. \\
& \left.+\cos \left((\Omega+\omega) t+\theta_{x}+\phi\right)\right) \\
a_{3}: & \frac{1}{4} X_{0}^{2}\left(2 \sin (\omega t+\phi)-\sin \left((2 \Omega+\omega) t+2 \theta_{x}\right.\right. \\
& \left.+\phi)+\sin \left((2 \Omega-\omega) t+2 \theta_{x}-\phi\right)\right) \\
a_{4}: & \frac{1}{8} X_{0}^{3}\left(\cos \left((3 \Omega+\omega) t+3 \theta_{x}+\phi\right)\right. \\
& -\cos \left((3 \Omega-\omega) t+3 \theta_{x}-\phi\right)-\cdots \\
& -3 \cos \left((\Omega+\omega) t+\theta_{x}+\phi\right) \\
& \left.+3 \cos \left((\Omega-\omega) t+\theta_{x}-\phi\right)\right)
\end{aligned}
$$

Eq. (7) illustrates the increasing complexity of the coefficient-functions even for simple polynomials of relatively low order. An approach attempting to extract the coefficients from the amplitudes of some spectral lines, has been used in the past for very simple cases [1, 2]. Previous attempts suffered from numerical difficulties therefore a more direct approach with superior numerical behavior and a much greater scope is developed below.

\subsection{Curve-fitting the coefficients of the amplitude function, $1 D$ and $2 D$ cases}

As the driving signals which determines the position of the laser-beam are known, we are able to use these signals $(x=\eta(t), y=\mu(t))$, in our calculations (in particular, when the driving signals are periodic or harmonic). Substituting the known time functions for $x$ and $y$ in Eq. (2), we can express the output of the laserinterferometer for every instance of time (time sample) as a weighted sum of known functions.

Here we have used $\eta_{j}=\eta\left(t_{j}\right), \mu_{j}=\mu\left(t_{j}\right)$ and $\phi$ is determined relative to the excitation signal.

As a large number of time samples is acquired, one can combine Eq. (8) for $t_{j} ; j=1 \ldots N$ to obtain.

Eq. (9) can be solved (in the least-squares sense) for the required coefficients $a_{1}, a_{2} \ldots a_{P}$.

For example, choosing Eq. (5), we obtain Eq. (10) for the one-dimensional case.

Eq. (10) can be directly solved and a continuous estimate of the vibration amplitude along a scanned line can be obtained in one step. The traditional usage of the laser-vibrometer requires measurements from a large number points where for each point, both the amplitude and phase should be curve fitted. In the age of the desktop PC, the bottleneck of the whole process is the time needed to gather the time samples rather than the curve-fitting operation. Using a standard PC the larger problems which need to be solved once (Eq. (10)) consume much less time than the point-wise process where tens to thousands of points are treated.

This saving in time becomes more significant for the $2 \mathrm{D}$ case as a single scan replaces a two-dimensional grid of measurements.

\section{An experimental example}

A simple experiment was set-up in order to demonstrate the proposed method. In this experiment (see 


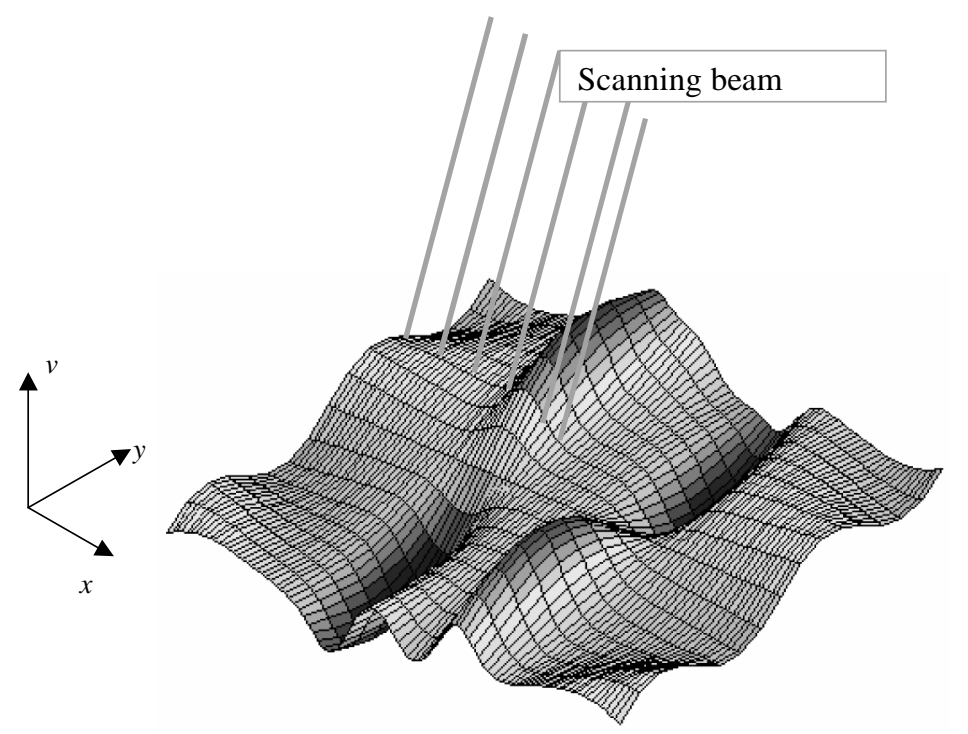

Fig. 1. Vibrating surface and scanning laser beam.

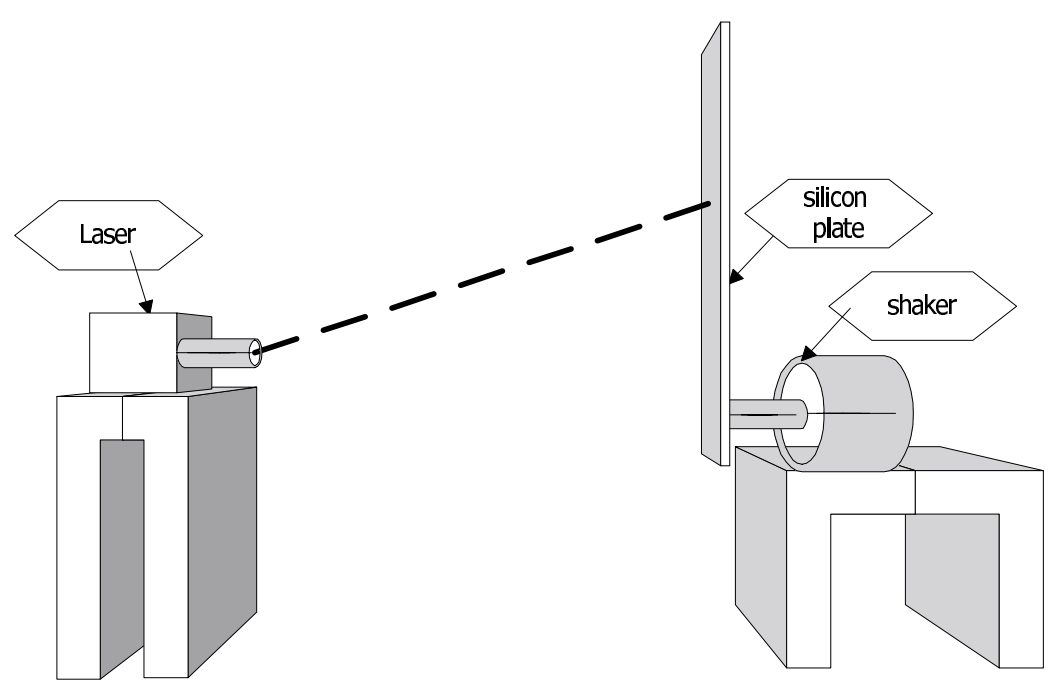

Fig. 2. Experimental set-up showing the excitation and measurement devices.

$$
\begin{aligned}
& V\left(t_{j}\right) \approx\left[f_{t}\left(\eta_{j}, \mu_{j}\right) f_{2}\left(\eta_{j}, \mu_{j}\right) f_{3}\left(\eta_{j}, \mu_{j}\right) \ldots\right]\left(\begin{array}{c}
a_{1} \\
a_{2} \\
a_{3} \\
\vdots
\end{array}\right) \sin \left(\omega t_{j}+\phi\right) \\
& \left(\begin{array}{c}
V\left(t_{1}\right) \\
\vdots \\
V\left(t_{N}\right)
\end{array}\right) \approx\left[\begin{array}{ccc}
f_{1}\left(\eta_{1}, \mu_{1}\right) \sin \left(\omega t_{1}+\phi\right) & \cdots & f_{P}\left(\eta_{1}, \mu_{1}\right) \sin \left(\omega t_{1}+\phi\right) \\
\vdots & & \vdots \\
f_{1}\left(\eta_{N}, \mu_{N}\right) \sin \left(\omega t_{N}+\phi\right) & \cdots & f_{P}\left(\eta_{N}, \mu_{N}\right) \sin \left(\omega t_{N}+\phi\right)
\end{array}\right]\left(\begin{array}{c}
a_{1} \\
a_{2} \\
a_{3} \\
\vdots
\end{array}\right)
\end{aligned}
$$

Fig. 2) a small silicon plate $(20 \times 100 \mathrm{~mm})$ was attached to a vibrating exciter. 


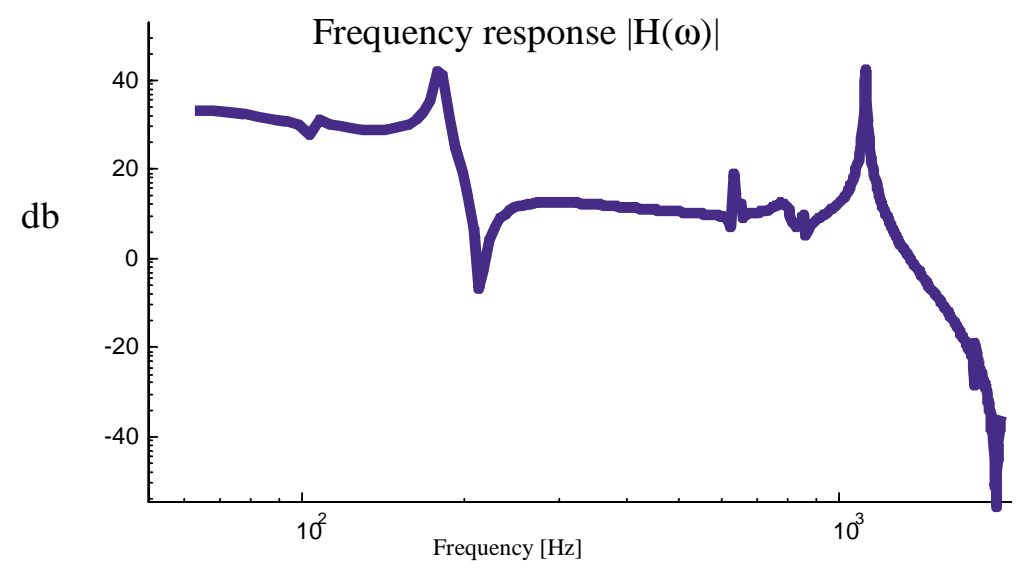

Fig. 3. Experimental set-up showing the excitation and measurement devices.

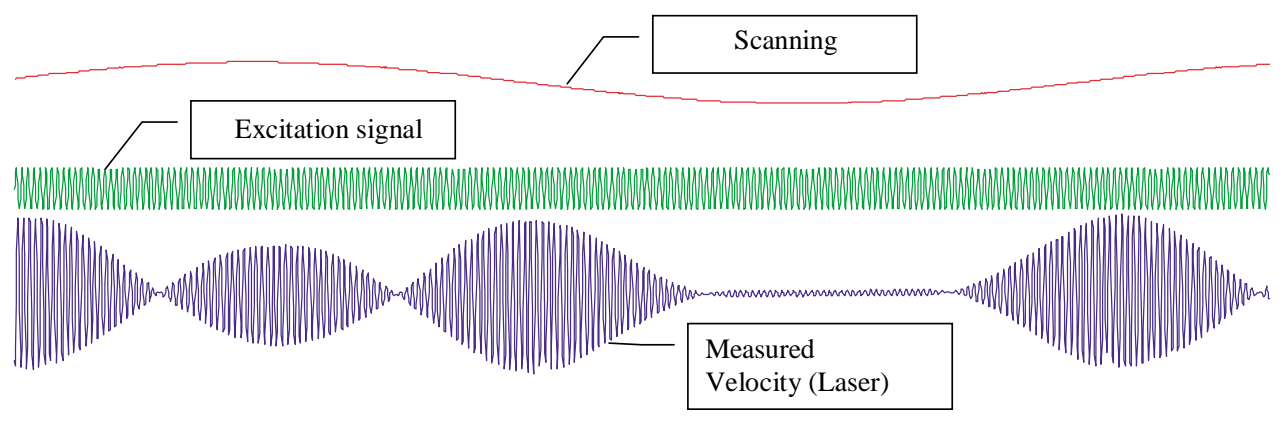

Time [s]

Fig. 4. A typical frequency response function of the vibrating plate.

$$
\left(\begin{array}{c}
V\left(t_{1}\right) \\
\vdots \\
V\left(t_{N}\right)
\end{array}\right) \approx\left[\begin{array}{cccc}
\sin \left(\omega t_{1}+\phi\right) & \eta\left(t_{1}\right) \sin \left(\omega t_{1}+\phi\right) & \eta^{2}\left(t_{1}\right) \sin \left(\omega t_{1}+\phi\right) & \eta^{P-1}\left(t_{1}\right) \sin \left(\omega t_{1}+\phi\right) \\
\vdots & & & \vdots \\
\sin \left(\omega t_{N}+\phi\right) & \eta\left(t_{N}\right) \sin \left(\omega t_{N}+\phi\right) & \eta^{2}\left(t_{N}\right) \sin \left(\omega t_{N}+\phi\right) & \eta^{P-1}\left(t_{N}\right) \sin \left(\omega t_{N}+\phi\right)
\end{array}\right]\left(\begin{array}{c}
a_{1} \\
a_{2} \\
a_{3} \\
\vdots
\end{array}\right)
$$

A stepped sine analysis was conducted while measuring at a single point close to the tip of the plate. The result is depicted in Fig. 3.

\subsection{Example of $1 D$ scanning}

By inspection of Fig. 3 it was decided to choose a frequency of excitation close to a natural frequency, in order to obtain a sufficiently large response. In this example $f=1116 \mathrm{~Hz}$ was chosen. A sinusoidal scanning function (see Eq. (5)) having a scanning frequency of $\Omega=6 \mathrm{~Hz}$ was used. The measured velocity signal in addition to the scanning command signal are depicted in Fig. 4.

As predicted by Eq. (7) the single frequency excitation resulted in a large number of harmonics. The spectrum of the measured laser response is depicted in Fig. 5.

Eq. (10) was used in the curve-fitting process where a 6th degree polynomial approximated the vibration amplitude. This parametric model produced a good fit of the measured response as compared to a fine pointwise scan and the fitted amplitude is shown below.

Figure 6 shows the fitted spatial amplitude as obtained by the parametric model in Eq. (2).

\subsection{Example of $2 D$ scanning}

In this case a $2 \mathrm{D}$ scan pattern was used in order to obtain the vibration amplitude of the vibrating surface. Using Eq. (10) once more with a suitable set of basis 


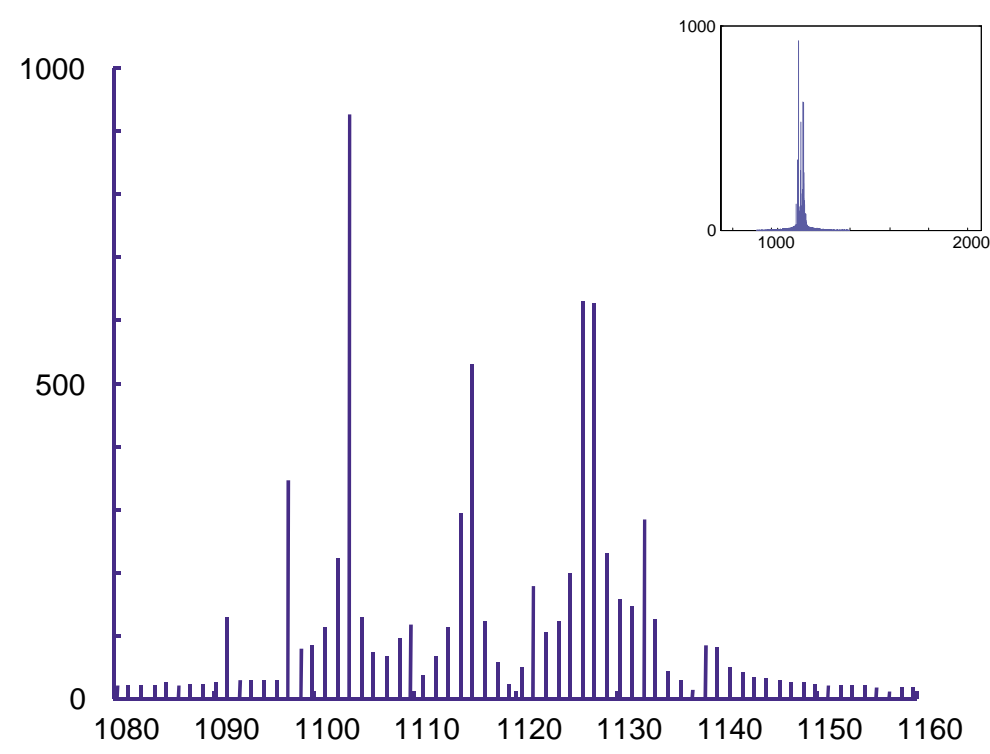

Fig. 5. The measured velocity the sine excitation and sinusoidal scanning.

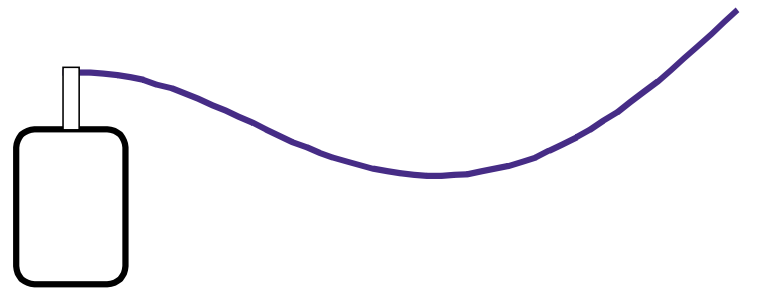

Fig. 6. Spectrum of measured signal while performing a continuous scan. Top left: broad frequency range, main figure: zoom around the excitation frequency.

functions, e.g.:

$$
f_{k}(x) \in\left\{1, x, y, x y, x^{2} y, x y^{2}, x^{3}, x^{4}, \ldots\right\}
$$

We are able to construct a new set of equations similar to Eq. (10) and solve for the required coefficients.

It can be observed that a good fit was obtained in the time-domain, as shown in Fig. 8.

The resulted amplitude function (see Fig. 9) resembled the Finite-element prediction and once more, due to the parametric model a smoothing operation is not required in this case.

\subsection{Numerical and signal processing issues}

It should be noted that the absence of certain harmonics singularity of Eq.(10) may result in spurious solutions. It was therefore found that a simple regularization [3] yields good results. Other important factors are (i) the effect of scanning: scanning introduces components at the scanning frequency and multiples these

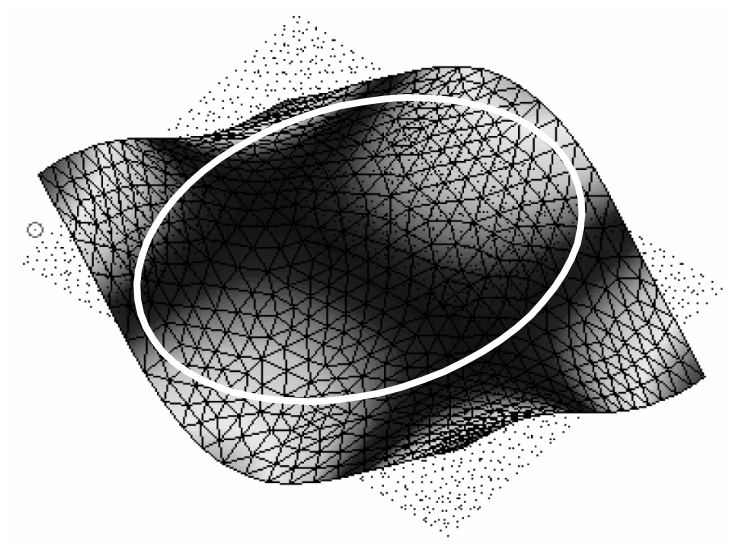

Fig. 7. Amplitude of plate vibrating at $f=1116 \mathrm{~Hz}$.

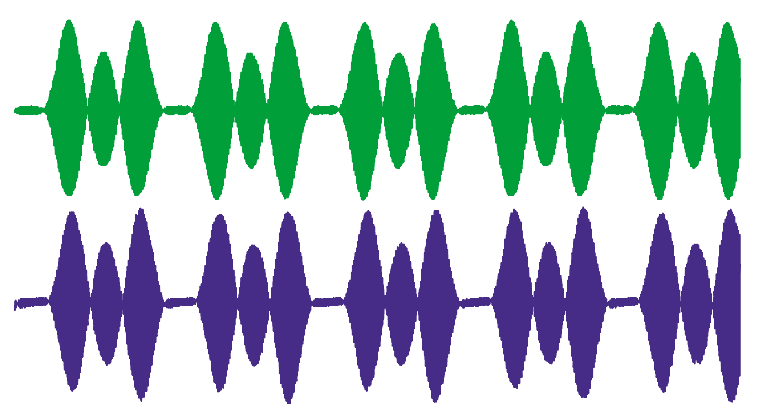

Fig. 8. Measured and curve-fitted (shifted by a constant) response signals.

frequencies should be eliminated or embedded into the parametric model (ii) The scanning mirros posses some 


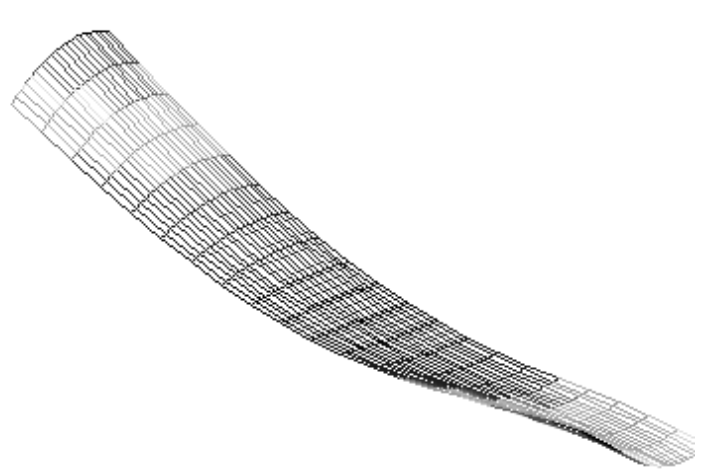

Fig. 9. Measured (curve-fitted) two-dimensional amplitude of vibration plate.

dynamical behavior, i.e. phase and amplitude distortions. If one uses a high scanning frequency these factors should also be incorporated in the model.

\section{Conclusions}

In this paper a direct mathematical approach to continuous laser scanning of vibrating surfaces was introduced. The method makes use of the advance in the computing power to replace more elaborate FFT- based methods that are using a discrete measurement grid. The usage of a parametric model, eliminates the problems associated with the Discrete Fourier transform, namely leakage. The method was proved successful when the amplitude of vibration was harmonic and sufficiently large to eliminate spurious effects such as laser scan frequency. The dynamics of the mirrors had to be taken into account and therefore a slow scanning rate was used and some phase-lag parameters beyond what is described here were used for different scan-conditions. Some more research is required to apply this method for in-situ conditions and some more progress in the performance of the scanning mechanism will eliminate some of the unnecessary difficulties.

\section{References}

[1] Bucher I., Schmiechen P., Robb D,A. and D.J. Ewins, A Laserbased measurement system for measuring the vibration on rotating discs, International conference on vibration measurement by Laser techniques, Ancona, Italy, October 1994.

[2] Stanbridge, A.B. and Ewins, D.J., Structural modal analysis using a scanning Laser Doppler vibrometer, International forum on aeroelasticity and structural dynamics, Manchester, 1995.

[3] Lawson C.L. and Hanson D.J., Solving least squares problems, Prentice-Hall, 1974. 

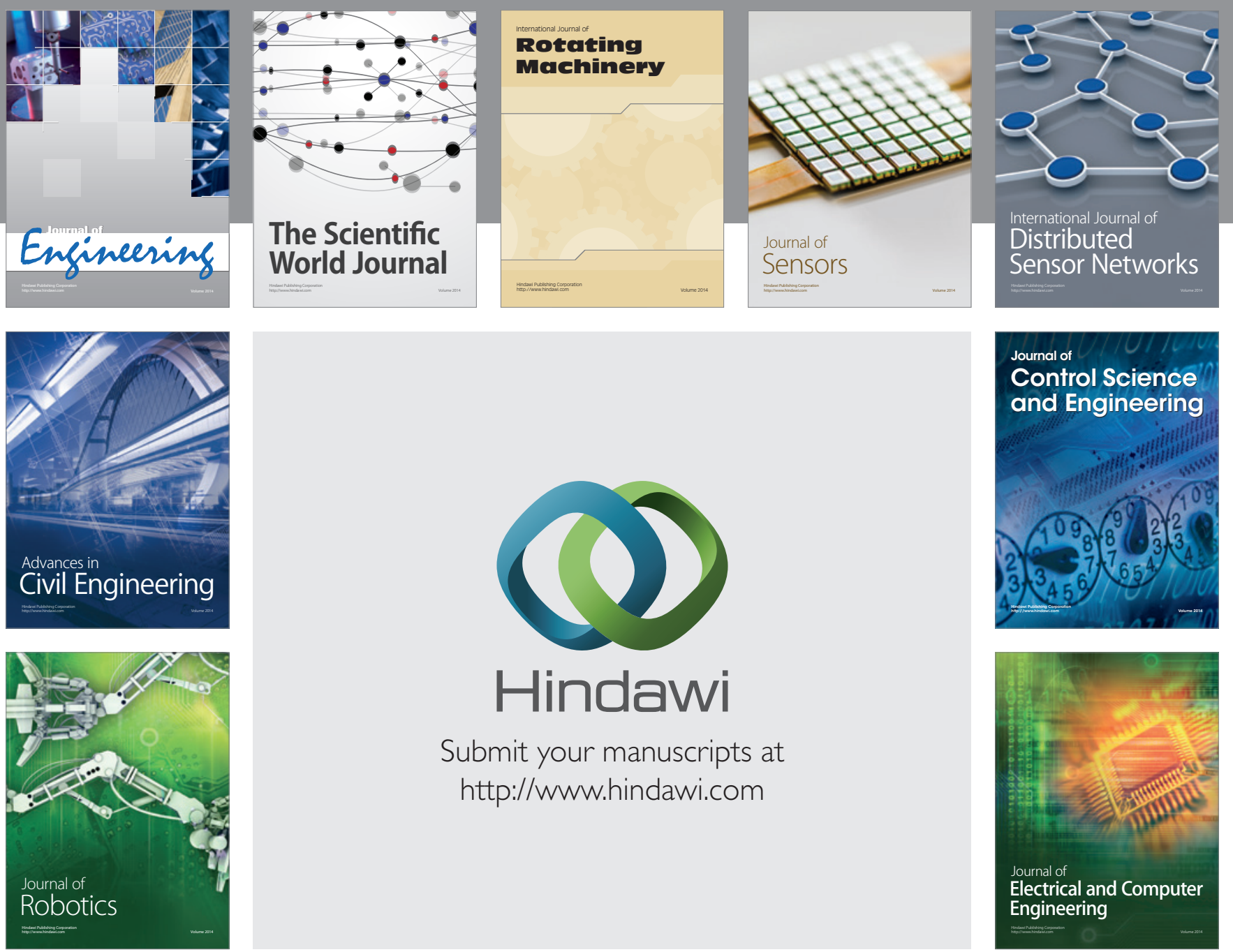

Submit your manuscripts at

http://www.hindawi.com
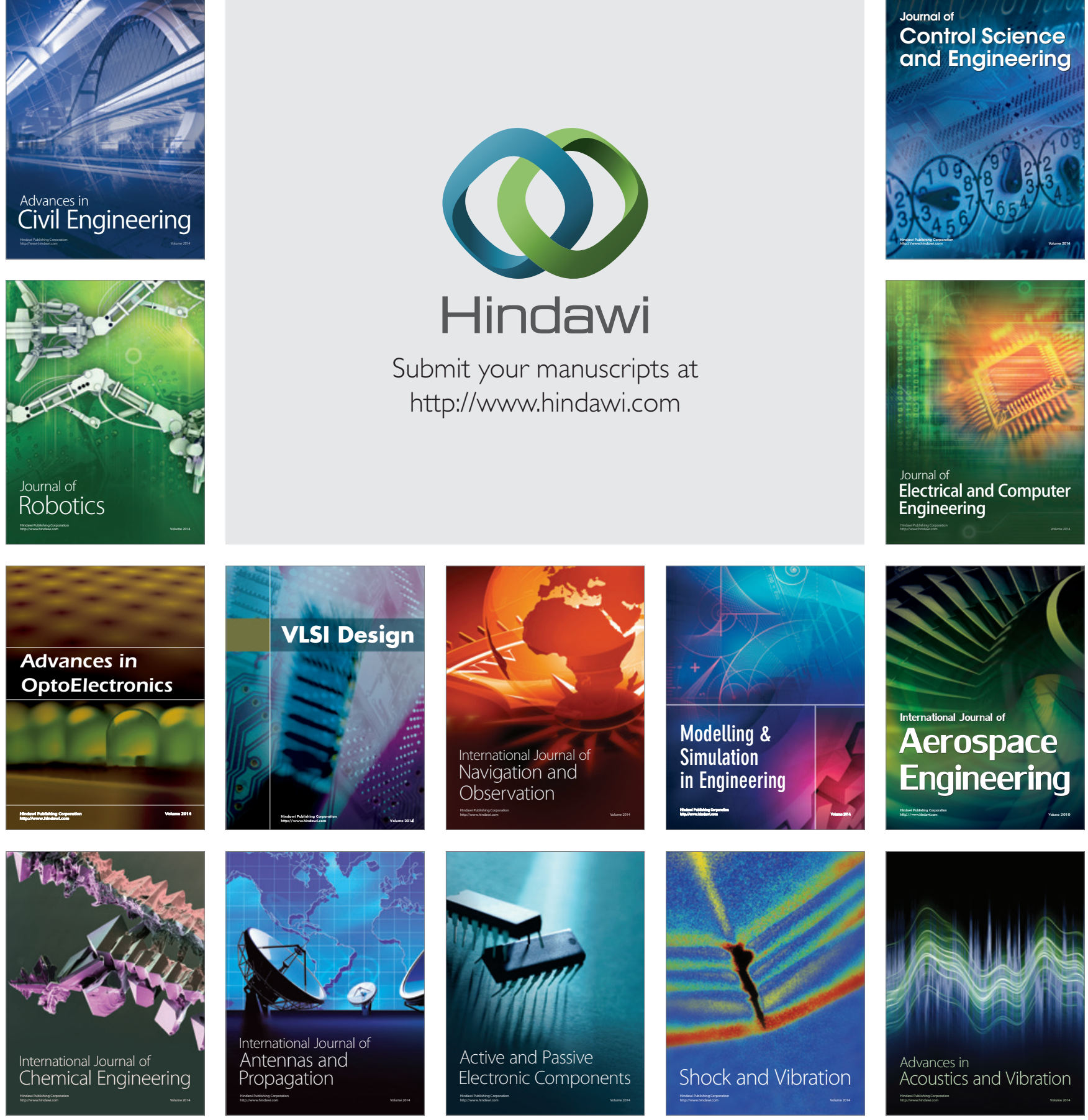